\title{
Erratum to: Holomorphic Linearization of Commuting Germs of Holomorphic Maps
}

\author{
Jasmin Raissy
}

Published online: 19 June 2012

(C) Mathematica Josephina, Inc. 2012

\section{Erratum to: J Geom Anal \\ DOI 10.1007/s12220-012-9316-2}

My original paper failed to cite the pioneering work of Laurent Stolovitch in the paper [1]. In particular, Theorem 1.3 can be seen as a consequence of Theorem 2.1 in [1].

\section{References}

1. Stolovitch, L.: Family of intersecting totally real manifolds of $\left(\mathbb{C}^{n}, 0\right)$ and CR-singularities $(2005$, preprint). arXiv:math/0506052v2

The online version of the original article can be found under doi:10.1007/s12220-012-9316-2.

J. Raissy $(\bowtie)$

Dipartimento di Matematica e Applicazioni, Università degli Studi di Milano Bicocca, Via Roberto Cozzi 53, 20125 Milano, Italy

e-mail: jasmin.raissy@unimib.it 